\title{
Wiener filter preprocessing for OFDM systems in the presence of both nonstationary and stationary phase noises
}

\author{
Ke Zhong*, Xia Lei and Shaoqian Li
}

\begin{abstract}
Statistics-based intercarrier interference (ICI) mitigation algorithm is proposed for orthogonal frequency division multiplexing systems in presence of both nonstationary and stationary phase noises. By utilizing the statistics of phase noise, which can be obtained from measurements or data sheets, a Wiener filter preprocessing algorithm for ICl mitigation is proposed. The proposed algorithm can be regarded as a performance-improving technique for the previous researches on phase noise cancelation. Simulation results show that the proposed algorithm can effectively mitigate $\mathrm{ICl}$ and lower the error floor, and therefore significantly improve the performances of previous researches on phase noise cancelation, especially in the presence of severe phase noise.
\end{abstract}

\section{Introduction}

Due to its high data rate transmission capability and its robustness to multipath delay spread, orthogonal frequency division multiplexing (OFDM) has been adopted in most parts of modern wireless communication systems, such as wireless local area networks [1], digital audio and video broadcasting [2], and so is OFDM being standardized for the future wireless communication systems, such as Worldwide Interoperability for Microwave Access [3] and 3GPP's Long-Term Evolution [4]. However, OFDM is known to be very sensitive to radio frequency frontend imperfections such as carrier frequency offset (CFO), in-phase and quadrature-phase (IQ) imbalance and phase noise. These imperfections, if not properly estimated and compensated, will severely degrade the performance of OFDM systems. CFO and IQ imbalance are constant over an OFDM symbol period and have widely been investigated and well solved in the literature [5-8]. However, unlike the constant CFO and IQ imbalance, phase noise is a stochastic process during an OFDM symbol period and therefore causes a greater challenging problem. This is because each OFDM sample in an OFDM symbol period suffers from different phase noise.

\footnotetext{
*Correspondence: zhongke@uestc.edu.cn

The National Key Laboratory of Science and Technology on Communications, University of Electronic Science and Technology of China, Chengdu 611731,
} P. R. China
The detrimental effect of phase noise on the performance of OFDM systems has extensively been studied in [9-11]. The effect of phase noise on OFDM is commonly categorized as common phase error (CPE) and intercarrier interference (ICI). Several techniques have been proposed to estimate and compensate for phase noise in OFDM systems [12-17]. More specifically, by trading the ICI as additional noise, Wu and Bar-Ness [12] use minimum mean square error criterion to cancel the CPE. By considering that phase noise can be modeled as a lowpass process, the authors of $[13,14]$ resort to estimate a few spectral components of phase noise. In particular, the ICI-cancelation scheme proposed in [13] stems from iterative detection principle, while [14] resorts to interpolation between the CPE estimates of two consecutive OFDM symbols in a noniterative way. Zou et al. $[15,16]$ propose to estimate a few phase noise components in the time domain and obtain the rest components by interpolation. In [17], each OFDM symbol is partitioned into several subblocks where phase noise process is assumed quasi-static over each subblock and therefore only the CPE of each subblock needs to be estimated.

In this article, we are concerned with ICI mitigation at the receiver side of OFDM systems using the statistics of phase noise, which can be obtained from measurements or data sheets (see $[9,10,13,18,19]$ and references therein). Based on the framework of Wiener filter, a Wiener filter

\section{Springer}

(c) 2013 Zhong et al: licensee Springer. This is an Open Access article distributed under the terms of the Creative Commons Attribution License (http://creativecommons.org/licenses/by/2.0), which permits unrestricted use, distribution, and reproduction in any medium, provided the original work is properly cited. 
preprocessing algorithm for ICI mitigation is proposed. The proposed algorithm performs directly on the received signal without changing the structure of conventional OFDM systems. Subsequently, the algorithms of previous researches on phase noise cancelation can be performed on the preprocessed received signal. Simulation results show that by utilizing the correlation inherently exists in phase noise, the proposed Wiener filter preprocessing algorithm can effectively mitigate the ICI and lower the error floor, and therefore significantly improve the performances of previous researches on phase noise cancelation, especially in the severe phase noise case.

The remainder of this article is organized as follows. Section 2 describes the OFDM system and phase noise models. The proposed Wiener filter preprocessing algorithm is presented in Section 3. Section 4 gives some simulation results that demonstrate the effectiveness of the proposed algorithm. Finally, conclusions are drawn in Section 5.

Notation: Vectors and matrices are boldface letters. A hat over a variable (e.g., $\hat{S}$ ) indicates an estimate of the variable. $\mathbb{E}\{\cdot\}$ denotes the expectation. Superscripts $[\cdot]^{T}$, $[\cdot]^{-1},[\cdot]^{H}$, and $[\cdot]^{*}$ denote the transpose, the matrix inversion, the Hermitian, and the complex conjugate operations, respectively. $\mathbf{I}_{N}$ is an identity matrix with dimension $N . \mathbf{0}_{N}$ denotes an $N \times 1$ vector whose elements are all zeros. The symbol $\circledast$ denotes convolution. $\mathfrak{R}\{\cdot\}$ and $\mathfrak{J}\{\cdot\}$ are the real and imaginary parts of the quantity in the brackets, respectively. $\delta(\cdot)$ denotes the Dirac delta function. The matrix $\mathbf{F}$ is the normalized discrete Fourier transform (DFT) matrix with the $(m, n)$ th element given by $[\mathbf{F}]_{m, n}=\frac{1}{\sqrt{N}} e^{-j 2 \pi \frac{m n}{N}}$.

\section{System model}

\subsection{OFDM model}

A model of the OFDM system in the presence of phase noise is depicted in Figure 1. In an OFDM system, the source data in the frequency domain $\mathbf{X}=$ $[X(0), X(1), \ldots, X(N-1)]^{T}$ is modulated onto $N$ parallel subcarriers to obtain the time domain signal $\mathbf{z}=$ $[z(0), z(1), \ldots, z(N-1)]^{T}=\mathbf{F}^{H} \mathbf{X}$. In general, the elements of $\mathbf{X}$ can be categorized into

$$
X(m)=\left\{\begin{array}{ll}
X_{d}(m) & \forall m \in I_{d} \\
X_{p}(m) & \forall m \in I_{p}
\end{array},\right.
$$

where $I_{d}$ is the index set of subcarriers allocated for data symbols with $N_{d}$ elements, and $I_{p}$ is the index set of subcarriers allocated for pilot symbols with $N_{p}$ elements, respectively. Notice that $N=N_{d}+N_{p}$. From (1), we have $\mathbf{X}=\mathbf{E}_{d} \mathbf{X}_{d}+\mathbf{E}_{p} \mathbf{X}_{p}$, where the $N \times N_{d}$ matrix $\mathbf{E}_{d}$ and $N \times N_{p}$ matrix $\mathbf{E}_{p}$ denote matrices collecting columns of $\mathbf{I}_{N}$ corresponding to $I_{d}$ and $I_{p}$, respectively, and $\mathbf{X}_{d}=\left[X_{d}(0), X_{d}(1), \ldots, X_{d}\left(N_{d}-1\right)\right]^{T}, \mathbf{X}_{p}=$
$\left[X_{p}(0), X_{p}(1), \ldots, X_{p}\left(N_{p}-1\right)\right]^{T}$ denote the data and pilot vectors, respectively. We assume that the OFDM subcarrier signals are mutually independent random variables with zero mean and variance $E_{s}$, i.e., $\mathbb{E}\left\{X\left(m_{1}\right) X^{*}\left(m_{2}\right)\right\}=E_{s} \delta\left(m_{1}-m_{2}\right)$. A cyclic prefix $(\mathrm{CP})$ with length $N_{\mathrm{cp}}$ longer than the delay spread of the channel is inserted at the beginning of each OFDM symbol to prevent intersymbol interference, i.e., the transmitted time domain signal is $\left[z\left(N-N_{c p}\right)\right.$, $\left.z\left(N-N_{c p}+1\right), \ldots, z(N-1), z(0), z(1), \ldots, z(N-1)\right]^{T}$.

At the receiver side, assuming perfect timing and frequency synchronization are achieved, the $n$th sample of the received signal is given by

$$
y(n)=e^{j \phi(n)} \sum_{l=0}^{L-1} h(l) z(n-l)+w(n),
$$

where $\phi(n)$ represents the phase noise, $w(n)$ 's are independent and identically distributed (i.i.d.) complex random variables with zero mean and variance $\sigma^{2}$, representing the contribution of additive white Gaussian noise. The coefficients $\{h(l)\}_{l=0}^{L-1}$ represent the equivalent multipath discrete-time channel impulse response (CIR) with length $L$, including transmit and receive filters, i.e., $h(l)=$ $\left.g^{T x}(t) \circledast h^{P r}(t) \circledast g^{R x}(t)\right|_{t=l T_{s}}$, where $g^{T x}(t), h^{P r}(t)$, and $g^{R x}(t)$ denote the pulse shaping filter at the transmitter, the impulse response of the propagation medium, and the shaping filter at the receiver, respectively, and $T_{s}$ is the sampling interval. We consider a slow fading frequency-selective channel where the CIR is assumed to remain constant during the transmission of one OFDM symbol period but can change randomly from symbol to symbol. The CIR is assumed perfectly known at the receiver. Powerful schemes for accurate channel estimation in the presence of phase noise have been proposed in [20-22]. The channel taps are assumed mutually independent and the correlation function of different taps is given by $\mathbb{E}\left\{h\left(l_{1}\right) h^{*}\left(l_{2}\right)\right\}=\sigma_{l_{1}}^{2} \delta\left(l_{1}-l_{2}\right)$, where $\sigma_{l}^{2}$ denotes the average power of the $l$ th tap and without loss of generality, we assume $\sum_{l=0}^{L-1} \sigma_{l}^{2}=1$.

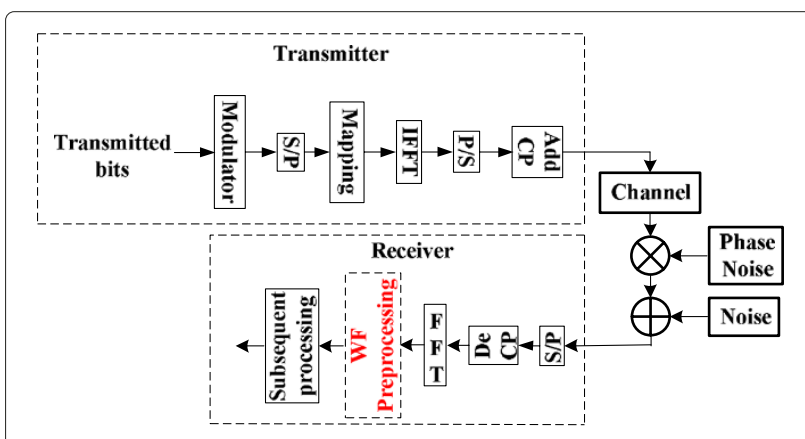

Figure 1 The OFDM system in the presence of phase noise. 
After discarding the $\mathrm{CP}$, the received signal after transforming back into the frequency domain is given by

$$
\begin{aligned}
Y(k) & =\frac{1}{\sqrt{N}} \sum_{n=0}^{N-1} y(n) e^{-j 2 \pi \frac{n k}{N}} \\
& =H(k) X(k) \underbrace{J(0)}_{\mathrm{CPE}}+\underbrace{\sum_{r=0, r \neq k}^{N-1} H(r) X(r) J(k-r)}_{\text {ICI }}+W(k) \\
& \triangleq \underbrace{S(k)}_{\text {the desired term }}+\underbrace{I(k)}_{\text {ICI }}+\underbrace{W(k)}_{\text {noise }},
\end{aligned}
$$

where $J(k)$, the DFT of the phase noise process, is given by

$$
J(k)=\frac{1}{N} \sum_{n=0}^{N-1} e^{j \phi(n)} e^{-j 2 \pi \frac{n k}{N}} .
$$

The DC coefficient $J(0)$ acts on all subcarriers as a CPE in (3) and the second term on the right-hand side of (3) represents ICI that results from higher order of $J(k)$. The channel frequency response $H(k)$ is given by

$$
H(k)=\sum_{l=0}^{L-1} h(l) e^{-j 2 \pi \frac{l k}{N}} .
$$

$W(k)$ represents the corresponding noise in the frequency domain. It is observed that the effect of phase noise, i.e., $\mathrm{CPE}$ and ICI, distorts the received signal $Y(k)$ in a multiplicative and additive manner, respectively. Due to the detrimental effect of phase noise on the performance of OFDM systems, the phase noise should be compensated for.

\subsection{Phase noise model}

\subsubsection{Nonstationary phase noise}

When the system is frequency locked, the resulting phase noise is slowly varying but not limited, and it is modeled as a zero-mean, nonstationary, infinite-power Wiener process. In this case, the phase noise is expressed as a free-running or Brownian process, i.e.,

$$
\phi(n)=\phi(n-1)+v(n), n=0,1, \ldots, N-1,
$$

where $v(n)$ is an i.i.d zero-mean Gaussian variable with variance $\sigma_{v}^{2}=2 \pi \beta T / N$, with $\beta$ representing the two-side $3-\mathrm{dB}$ linewidth of phase noise, $\beta$ T representing the phase noise rate $[9,10]$ and $T$ being the OFDM symbol period. In the case of perfect synchronization at the beginning of the OFDM symbol, $\phi(-1)=0$ and therefore, (6) can be expressed alternatively as

$$
\phi(n)=\sum_{m=0}^{n} v(m), n=0,1, \ldots, N-1 .
$$

\subsubsection{Stationary phase noise}

When the system is phase-locked, the resulting phase noise is low and modeled as a zero-mean, stationary, finite-power stochastic process. For a classical model of stationary phase noise, $\phi(n)$ is modeled as a stationary Gaussian process with zero mean and a specified power spectrum density $[13,18,19]$.

\section{The proposed Wiener filter preprocessing algorithm}

It is observed from (3) that we wish to estimate the desired term $S(k)$, from $Y(k), k=0,1, \ldots, N-1$. In the framework of Wiener filter, the estimate of $S(k)$, i.e., $\hat{S}(k)$, is determined by estimating a set of coefficients $\left\{\gamma_{k i}\right\}_{i=0}^{N}$, in order to minimize the estimation mean square error (MSE) as

$$
\left\{\hat{\gamma}_{k i, \mathrm{opt}}\right\}_{i=0}^{N}=\operatorname{argmin}_{\left\{\gamma_{k i}\right\}_{i=0}^{N}} \mathbb{E}\left\{|S(k)-\hat{S}(k)|^{2}\right\}
$$

$$
\text { s.t. } \hat{S}(k)=\sum_{i=0}^{N-1} \gamma_{k i} Y(i)+\gamma_{k N}
$$

where $\gamma_{k N}$ is a bias term that allows for nonzero means of $S(k)$ and $Y(k), k=0,1, \ldots, N-1$. Substituting (9) into (8) and setting the first derivative of the resulting (8) with respect to $\gamma_{k N}$ to zero, we obtain the optimal estimate of $\gamma_{k N}$ as

$$
\hat{\gamma}_{k N, \text { opt }}=\mathbb{E}\{S(k)\}-\sum_{i=0}^{N-1} \gamma_{k i} \mathbb{E}\{Y(i)\} .
$$

Substituting (10) into (8), the MSE can be expressed as

$$
\begin{aligned}
\mathbb{E}\left\{|S(k)-\hat{S}(k)|^{2}\right\}= & \Upsilon_{k}^{H} \Sigma_{\mathbf{Y Y}} \Upsilon_{k}-2 \Re\left\{\Upsilon_{k}^{H} \Sigma_{\mathbf{Y} S(k)}\right\} \\
& +\Sigma_{S(k) S(k)},
\end{aligned}
$$

where $\Upsilon_{k}=\left[\gamma_{k 0}, \gamma_{k 1}, \ldots, \gamma_{k N-1}\right]^{T}, \Sigma_{\mathbf{Y Y}}$ is the covariance matrix of $\mathbf{Y}=[Y(0), Y(1), \ldots, Y(N-1)]^{T}, \Sigma_{\mathbf{Y} S(k)}$ is the cross-covariance vector of $\mathbf{Y}$ and $S(k), \Sigma_{S(k) S(k)}$ is the variance of $S(k)$. Setting the first derivative of $(11)$ with respect to $\Upsilon_{k}$ to zero, we obtain the optimal estimate of $\Upsilon_{k}$ as

$$
\hat{\Upsilon}_{k, o p t}=\Sigma_{\mathbf{Y Y}}^{-1} \Sigma_{\mathbf{Y} S(k)}
$$

Substituting (10) and (12) into (9), the optimal estimate of $S(k)$ can thus be obtained as

$$
\hat{S}_{\text {opt }}(k)=\mathbb{E}\{S(k)\}+\Sigma_{\mathbf{Y} S(k)}^{H} \Sigma_{\mathbf{Y Y}}^{-1}(\mathbf{Y}-\mathbb{E}\{\mathbf{Y}\}) .
$$

Stacking all $N$ subcarriers, the optimal estimate of $\mathbf{S}=[S(0), S(1), \ldots, S(N-1)]^{T}$ can be written in matrix form as 


$$
\begin{aligned}
\hat{\mathbf{S}}_{\mathrm{opt}} & =\left[\begin{array}{c}
\mathbb{E}\{S(0)\} \\
\mathbb{E}\{S(1)\} \\
\vdots \\
\mathbb{E}\{S(N-1)\}
\end{array}\right]+\left[\begin{array}{c}
\boldsymbol{\Sigma}_{\mathbf{Y} S(0)}^{H} \\
\boldsymbol{\Sigma}_{\mathbf{Y} S(1)}^{H} \\
\vdots \\
\boldsymbol{\Sigma}_{\mathbf{Y} S(N-1)}^{H}
\end{array}\right] \boldsymbol{\Sigma}_{\mathbf{Y Y}}^{-1}(\mathbf{Y}-\mathbb{E}\{\mathbf{Y}\}) \\
& \triangleq \mathbb{E}\{\mathbf{S}\}+\Sigma_{\mathbf{S Y}} \boldsymbol{\Sigma}_{\mathbf{Y Y}}^{-1}(\mathbf{Y}-\mathbb{E}\{\mathbf{Y}\})
\end{aligned}
$$

where

$$
\begin{aligned}
\Sigma_{\mathbf{S Y}} & =\mathbb{E}\left\{(\mathbf{S}-\mathbb{E}\{\mathbf{S}\})(\mathbf{Y}-\mathbb{E}\{\mathbf{Y}\})^{H}\right\} \\
& =\mathbb{E}\left\{\mathbf{S} \mathbf{Y}^{H}\right\}-\mathbb{E}\{\mathbf{S}\} \mathbb{E}\left\{\mathbf{Y}^{H}\right\},
\end{aligned}
$$

and

$$
\begin{aligned}
\boldsymbol{\Sigma}_{\mathbf{Y Y}} & =\mathbb{E}\left\{(\mathbf{Y}-\mathbb{E}\{\mathbf{Y}\})(\mathbf{Y}-\mathbb{E}\{\mathbf{Y}\})^{H}\right\} \\
& =\mathbb{E}\left\{\mathbf{Y} \mathbf{Y}^{H}\right\}-\mathbb{E}\{\mathbf{Y}\} \mathbb{E}\left\{\mathbf{Y}^{H}\right\} .
\end{aligned}
$$

In (15), $\mathbb{E}\left\{\mathbf{S Y}{ }^{H}\right\}$ is derived as

$$
\begin{aligned}
\mathbb{E}\left\{\mathbf{S Y}^{H}\right\} & =\mathbb{E}\left\{\mathbf{S}(\mathbf{S}+\mathbf{I}+\mathbf{W})^{H}\right\} \\
& =\Phi_{\mathbf{S S}}+\Phi_{\mathbf{S I}},
\end{aligned}
$$

and in (16), $\mathbb{E}\left\{\mathbf{Y Y}{ }^{H}\right\}$ is derived as

$$
\begin{aligned}
\mathbb{E}\left\{\mathbf{Y Y}^{H}\right\} & =\mathbb{E}\left\{(\mathbf{S}+\mathbf{I}+\mathbf{W})(\mathbf{S}+\mathbf{I}+\mathbf{W})^{H}\right\} \\
& =\Phi_{\mathbf{S S}}+\Phi_{\mathbf{I I}}+\Phi_{\mathbf{S I}}+\Phi_{\mathbf{S I}}^{H}+\Phi_{\mathbf{W W}},
\end{aligned}
$$

where we have assumed that the desired term $\mathbf{S}$ and the ICI $\mathbf{I}=[I(0), I(1), \ldots, I(N-1)]^{T}$ are independent of the noise $\mathbf{W}=[W(0), W(1), \ldots, W(N-1)]^{T}$, and $\Phi_{\mathbf{P Q}}$ represents the correlation function between $\mathbf{P}$ and $\mathbf{Q}$.

We now derive each term required to compute $\hat{\mathbf{S}}_{\text {opt }}$ in (14). From (3), the elements of $\Phi_{S S}$ in (17) and (18) can be obtained as

$$
\begin{aligned}
\mathbb{E}\left\{S\left(k_{1}\right) S^{*}\left(k_{2}\right)\right\}= & \mathbb{E}\left\{\left(H\left(k_{1}\right) X\left(k_{1}\right) J(0)\right)\left(H\left(k_{2}\right) X\left(k_{2}\right) J(0)\right)^{*}\right\} \\
= & \mathbb{E}\left\{H\left(k_{1}\right) H^{*}\left(k_{2}\right)\right\} \\
& \times \mathbb{E}\left\{X\left(k_{1}\right) X^{*}\left(k_{2}\right)\right\} \mathbb{E}\left\{J(0) J^{*}(0)\right\},
\end{aligned}
$$

where the fact that the transmit signal, the wireless channel, and the phase noise are independent from each other has been used. Using (5), the correlation function $\mathbb{E}\left\{H\left(k_{1}\right) H^{*}\left(k_{2}\right)\right\}$ can be derived as

$$
\begin{aligned}
\mathbb{E}\left\{H\left(k_{1}\right) H^{*}\left(k_{2}\right)\right\}= & \mathbb{E}\left\{\left(\sum_{l_{1}=0}^{L-1} h\left(l_{1}\right) e^{-j 2 \pi \frac{l_{1} k_{1}}{N}}\right)\right. \\
& \left.\times\left(\sum_{l_{2}=0}^{L-1} h\left(l_{2}\right) e^{-j 2 \pi \frac{l_{2} k_{2}}{N}}\right)^{*}\right\} \\
= & \sum_{l_{1}=0}^{L-1} \sum_{l_{2}=0}^{L-1} \mathbb{E}\left\{h\left(l_{1}\right) h^{*}\left(l_{2}\right)\right\} e^{-j 2 \pi \frac{\left(l_{1} k_{1}-l_{2} k_{2}\right)}{N}} \\
= & \sum_{l=0}^{L-1} \sigma_{l}^{2} e^{-j 2 \pi l \frac{\left(k_{1}-k_{2}\right)}{N}} .
\end{aligned}
$$

Therefore, substituting (20) into (19) we obtain that if $k_{1} \neq k_{2}$,

$$
\begin{aligned}
\mathbb{E}\left\{S\left(k_{1}\right) S^{*}\left(k_{2}\right)\right\} & =E_{s} \mathbb{E}\left\{J(0) J^{*}(0)\right\} \sum_{l=0}^{L-1} \sigma_{l}^{2} e^{-j 2 \pi l \frac{\left(k_{1}-k_{2}\right)}{N}} \delta\left(k_{1}-k_{2}\right) \\
& =0
\end{aligned}
$$

and if $k_{1}=k_{2}$,

$$
\mathbb{E}\left\{S\left(k_{1}\right) S^{*}\left(k_{1}\right)\right\}=E_{S} \mathbb{E}\left\{J(0) J^{*}(0)\right\} .
$$

Using (3) the elements of $\Phi_{\text {II }}$ in (18) can be derived as

$$
\begin{aligned}
\mathbb{E}\left\{I\left(k_{1}\right) I^{*}\left(k_{2}\right)\right\}= & \mathbb{E}\left\{\left(\sum_{r_{1}=0, r_{1} \neq k_{1}}^{N-1} H\left(r_{1}\right) X\left(r_{1}\right) J\left(k_{1}-r_{1}\right)\right)\right. \\
& \left.\times\left(\sum_{r_{2}=0, r_{2} \neq k_{2}}^{N-1} H\left(r_{2}\right) X\left(r_{2}\right) J\left(k_{2}-r_{2}\right)\right)^{*}\right\},
\end{aligned}
$$

and therefore, if $k_{1} \neq k_{2}$,

$$
\mathbb{E}\left\{I\left(k_{1}\right) I^{*}\left(k_{2}\right)\right\}=E_{s} \sum_{r=0, r \neq k_{1}, k_{2}}^{N-1} \mathbb{E}\left\{J\left(k_{1}-r\right) J^{*}\left(k_{2}-r\right)\right\},
$$

and if $k_{1}=k_{2}$,

$\mathbb{E}\left\{I\left(k_{1}\right) I^{*}\left(k_{1}\right)\right\}=E_{s} \sum_{r=0, r \neq k_{1}}^{N-1} \mathbb{E}\left\{J\left(k_{1}-r\right) J^{*}\left(k_{1}-r\right)\right\}$.

Similarly, the elements of $\Phi_{S I}$ in (17) and (18) can be derived as

$$
\begin{aligned}
\mathbb{E}\left\{S\left(k_{1}\right) I^{*}\left(k_{2}\right)\right\}=\mathbb{E}\left\{\left(H\left(k_{1}\right) X\left(k_{1}\right) J(0)\right)\right. \\
\left.\times\left(\sum_{r=0, r \neq k_{2}}^{N-1} H(r) X(r) J\left(k_{2}-r\right)\right)^{*}\right\},
\end{aligned}
$$

and therefore, if $k_{1} \neq k_{2}$

$$
\mathbb{E}\left\{S\left(k_{1}\right) I^{*}\left(k_{2}\right)\right\}=E_{s} \mathbb{E}\left\{J(0) J^{*}\left(k_{2}-k_{1}\right)\right\},
$$

and if $k_{1}=k_{2}$

$$
\mathbb{E}\left\{S\left(k_{1}\right) I^{*}\left(k_{1}\right)\right\}=0 .
$$

It is noted from (19)-(28) that the correlation function $\mathbb{E}\left\{J\left(k_{1}\right) J^{*}\left(k_{2}\right)\right\}$ plays a pivotal role in the computation of $\Phi_{\text {SS }}, \Phi_{\text {II }}$, and $\Phi_{\text {SI }}$. 
Using (4), the correlation function $\mathbb{E}\left\{J\left(k_{1}\right) J^{*}\left(k_{2}\right)\right\}$ can be derived as

$$
\begin{aligned}
\mathbb{E}\left\{J\left(k_{1}\right) J^{*}\left(k_{2}\right)\right\}=\frac{1}{N^{2}} \mathbb{E}\left\{\left(\sum_{n_{1}=0}^{N-1} e^{j \phi\left(n_{1}\right)} e^{-j 2 \pi \frac{n_{1} k_{1}}{N}}\right)\right. \\
\left.\quad \times\left(\sum_{n_{2}=0}^{N-1} e^{j \phi\left(n_{2}\right)} e^{-j 2 \pi \frac{n_{2} k_{2}}{N}}\right)^{*}\right\} \\
=\frac{1}{N^{2}} \sum_{n_{1}=0}^{N-1} \sum_{n_{2}=0}^{N-1} \mathbb{E}\left\{e^{j\left(\phi\left(n_{1}\right)-\phi\left(n_{2}\right)\right)}\right\} e^{-j 2 \pi \frac{\left(n_{1} k_{1}-n_{2} k_{2}\right)}{N}} .
\end{aligned}
$$

\subsection{Nonstationary phase noise}

Substituting (7) into (29), we obtain

$$
\begin{aligned}
\mathbb{E}\left\{J\left(k_{1}\right) J^{*}\left(k_{2}\right)\right\}= & \frac{1}{N^{2}} \sum_{n_{1}=0}^{N-1} \sum_{n_{2}=0}^{N-1} \\
& \mathbb{E}\left\{e^{j\left(\sum_{m_{1}=0}^{n_{1}} v\left(m_{1}\right)-\sum_{m_{2}=0}^{n_{2}} v\left(m_{2}\right)\right)}\right\} \\
& \times e^{-j 2 \pi \frac{\left(n_{1} k_{1}-n_{2} k_{2}\right)}{N}} \\
= & \frac{1}{N^{2}} \sum_{n_{1}=0}^{N-1} \sum_{n_{2}=0}^{N-1} \\
& \mathbb{E}\left\{e^{j \operatorname{sgn}\left(n_{1}-n_{2}\right)\left(\sum_{m=0}^{\left|n_{1}-n_{2}\right|-1} v(m)\right)}\right\} \\
& \times e^{-j 2 \pi \frac{\left(n_{1} k_{1}-n_{2} k_{2}\right)}{N}},
\end{aligned}
$$

where $\operatorname{sgn}(n)$ represents the sign operation, i.e.,

$$
\operatorname{sgn}(n)= \begin{cases}1, & n>0 \\ 0, & n \leq 0\end{cases}
$$

Since $v(n)$ is an i.i.d zero-mean Gaussian variable with variance $\sigma_{v}^{2},\left\{\operatorname{sgn}\left(n_{1}-n_{2}\right) v(m)\right\}_{m=0}^{\left|n_{1}-n_{2}\right|-1}$ are also i.i.d. Gaussian variable with zero mean and variance given by

$$
\Sigma_{\left(\operatorname{sgn}\left(n_{1}-n_{2}\right) v(m)\right)\left(\operatorname{sgn}\left(n_{1}-n_{2}\right) v(m)\right)}=\mathbb{E}\left\{\left(\operatorname{sgn}\left(n_{1}-n_{2}\right)\right)^{2}(v(m))^{2}\right\}
$$

$$
=\sigma_{v}^{2} \text {. }
$$

Therefore, the variance of $\operatorname{sgn}\left(n_{1}-n_{2}\right)\left(\sum_{m=0}^{\left|n_{1}-n_{2}\right|-1} v(m)\right)$ can be derived as

$$
\begin{aligned}
& \left.\Sigma_{\left(\operatorname{sgn}\left(n_{1}-n_{2}\right)\right.}\left(\sum_{m=0}^{\left|n_{1}-n_{2}\right|-1} v(m)\right)\right)\left(\operatorname{sgn}\left(n_{1}-n_{2}\right)\left(\sum_{m=0}^{\left|n_{1}-n_{2}\right|-1} v(m)\right)\right) \\
& =\left|n_{1}-n_{2}\right| \sigma_{v}^{2} .
\end{aligned}
$$

Notice that for a Gaussian variable $\alpha$ with mean $\mu$ and variance $\psi^{2}$, its characteristic function is given by [23]

$$
\mathbb{E}\left\{e^{j t \alpha}\right\}=e^{j \mu t-\frac{\psi^{2} t^{2}}{2}} .
$$

Substituting (33) into (34) and letting $\mu=0$ and $t=1$, we obtain

$$
\mathbb{E}\left\{e^{j \operatorname{sgn}\left(n_{1}-n_{2}\right)\left(\sum_{m=0}^{\left|n_{1}-n_{2}\right|-1} v(m)\right)}\right\}=e^{-\frac{\left|n_{1}-n_{2}\right| \sigma_{V}^{2}}{2}} .
$$

Substituting (35) into (30), the correlation function $\mathbb{E}\left\{J\left(k_{1}\right) J^{*}\left(k_{2}\right)\right\}$ for the nonstationary phase noise case can be obtained.

\subsection{Stationary phase noise}

Since $\phi(n)$ is modeled as a stationary Gaussian process with zero mean, $\phi\left(n_{1}\right)-\phi\left(n_{2}\right)$ is also a stationary Gaussian process with zero mean and variance given by

$$
\begin{aligned}
\Sigma_{\left(\phi\left(n_{1}\right)-\phi\left(n_{2}\right)\right)\left(\phi\left(n_{1}\right)-\phi\left(n_{2}\right)\right)} & =\mathbb{E}\left\{\left(\left(\phi\left(n_{1}\right)-\phi\left(n_{2}\right)\right)^{2}\right\}\right. \\
& =2 \Phi_{\phi(0) \phi(0)}-2 \Phi_{\phi\left(n_{1}-n_{2}\right) \phi\left(n_{1}-n_{2}\right) .}
\end{aligned}
$$

Therefore, substituting (36) into (34) and letting $\mu=0$ and $t=1$, we obtain

$$
\mathbb{E}\left\{e^{j\left(\phi\left(n_{1}\right)-\phi\left(n_{2}\right)\right)}\right\}=e^{\Phi_{\phi\left(n_{1}-n_{2}\right) \phi\left(n_{1}-n_{2}\right)}-\Phi_{\phi(0) \phi(0)}} .
$$

Similar to the nonstationary one, substituting (37) into (29), the correlation function $\mathbb{E}\left\{J\left(k_{1}\right) J^{*}\left(k_{2}\right)\right\}$ for the stationary phase noise case can be obtained.

Finally, considering that

$$
\begin{aligned}
\mathbb{E}\{S(k)\} & =\mathbb{E}\{H(k)\} \mathbb{E}\{X(k)\} \mathbb{E}\{J(0)\} \\
& =0
\end{aligned}
$$

and

$$
\begin{aligned}
\mathbb{E}\{Y(k)\}= & \mathbb{E}\{H(k)\} \mathbb{E}\{X(k)\} \mathbb{E}\{J(0)\} \\
& +\sum_{r=0, r \neq k}^{N-1} \mathbb{E}\{H(r)\} \mathbb{E}\{X(r)\} \mathbb{E}\{J(k-r)\}+\mathbb{E}\{W(k)\} \\
= & 0
\end{aligned}
$$

we have for $\mathbb{E}\{\mathbf{S}\}$ in (15) as

$$
\mathbb{E}\{\mathbf{S}\}=\mathbf{0}_{N},
$$

and for $\mathbb{E}\{\mathbf{Y}\}$ in (15) and (16) as

$$
\mathbb{E}\{\mathbf{Y}\}=\mathbf{0}_{N}
$$

Since the FFT does not change the noise distribution, $\Phi_{\mathbf{W W}}$ in (18) is given by

$$
\Phi_{\mathbf{W W}}=\sigma^{2} \mathbf{I}_{N} \text {. }
$$

In summary, substituting the derived results (21) and (22), (24) and (25), (27) and (28), (40)-(42) into (14), the optimal estimate $\hat{\mathbf{S}}_{\text {opt }}$ can be obtained. Notice that for the nonstationary phase noise case, (30) and (35) should be adopted; for the stationary phase noise case, (29) and (37) should be adopted.

It is noted that without changing the structure of conventional OFDM systems, the proposed Wiener filter preprocessing algorithm is based on the statistics of phase 
noise (which can be obtained from measurements or data sheets) and performs directly on the received signal $\mathbf{Y}$ (see Figure 1 for illustration). Subsequently, the algorithms of previous researches on phase noise cancelation (e.g., [1217]) can be performed based on the preprocessed received signal $\hat{\mathbf{S}}_{\text {opt }}$ instead of on the received signal $\mathbf{Y}$. We will show in the next section that by utilizing the correlation inherently exists in phase noise process, the proposed Wiener filter preprocessing algorithm can effectively mitigate the ICI which results from phase noise and lower the error floor, and therefore significantly improve the performances of previous researches phase noise cancelation, especially in severe phase noise case.

\section{Simulation results and discussions}

In this section, the performance of the proposed Wiener filter preprocessing algorithm for ICI mitigation is demonstrated by Monte Carlo simulations. In the simulations, each OFDM symbol has 128 subcarriers $(N=$ 128) and communicates over a bandwidth of $20 \mathrm{MHz}$. The sampling interval $T_{s}$ is thus $50 \mathrm{~ns}$. The data are modulated by 16QAM modulation. The channel has three taps $(L=3)$ with an exponential power delay profile, namely $\sigma_{l}^{2}=\exp (-\kappa l)((1-\exp (-\kappa)) /(1-\exp (-\kappa L))), l=$ $0,1, \ldots, L-1$ with $\kappa=1 / 3$. Each tap coefficient follows a complex Gaussian distribution. Fourteen equally spaced OFDM subcarriers are allocated to pilot symbols, i.e., $N_{p}=14$ and $N_{d}=114$. This means that roughly $10.94 \%$ of the subcarriers are occupied by pilots. The pilots are used for the CPE estimation and cancelation algorithm given in [12] (marked as "conventional [12]" in
Figures 2, 3, 4, and 5). The pilots are also used for the ICIcancelation algorithm given in [13] with ICI correction of order 3 (marked as "conventional [13]" in Figures 2, 3, 4, and 5). The performance of $[12,13]$ preprocessed by our proposed Wiener filter preprocessing algorithm is marked as "proposed [12]" and "proposed [13]" in Figures 2, 3, 4, and 5.

For the nonstationary phase noise case, it can be seen from Figure 2 that if the detrimental effect of phase noise, i.e., CPE and ICI, are left uncompensated (marked as "no phase noise correction" in Figures 2, 3, 4, and 5), the performance is totally unacceptable for both cases where phase noise rate are 0.1 and 0.01 .

For the case that phase noise rate is 0.01 (dashed lines in Figure 2), the CPE dominates the effect of phase noise. Therefore, as can be seen from Figure 2, the performance can significantly be improved if only CPE is compensated. If [12] is preprocessed by our proposed Wiener filter preprocessing algorithm, there is a minor performance improvement for "proposed [12]" compared to "conventional [12]", as can be observed from Figure 2. Although ICI is small in this case, if ICI is also mitigated, a minor performance improvement can be obtained especially in high SNR region, where the decision on the transmitted symbols are more accurate than that in low SNR region and therefore, the ICI can be more accurately estimated and canceled according to [13]. It is observed from Figure 2 that the performance of "proposed [13]" is almost the same as that of the "conventional [13]" (only with minor improvement) for the reason that ICI is small in this case.

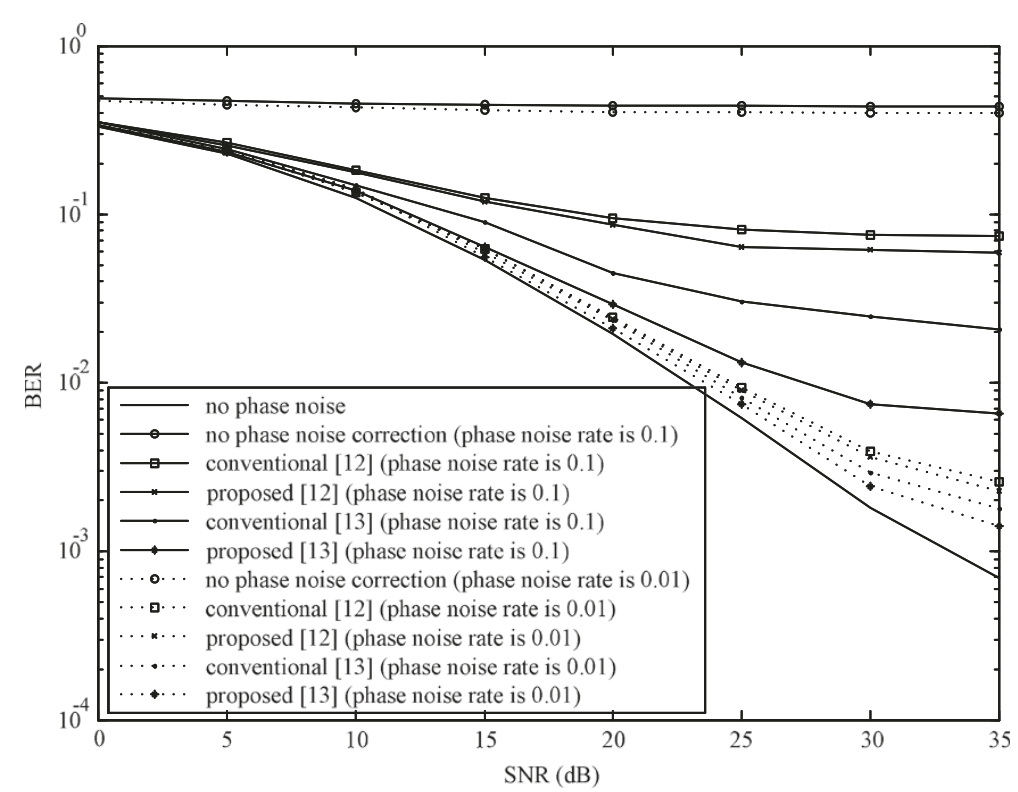

Figure 2 BER performance: nonstationary phase noise case. BER versuS SNR for nonstationary phase noise, where the phase noise rates are 0.1 and 0.01 . 


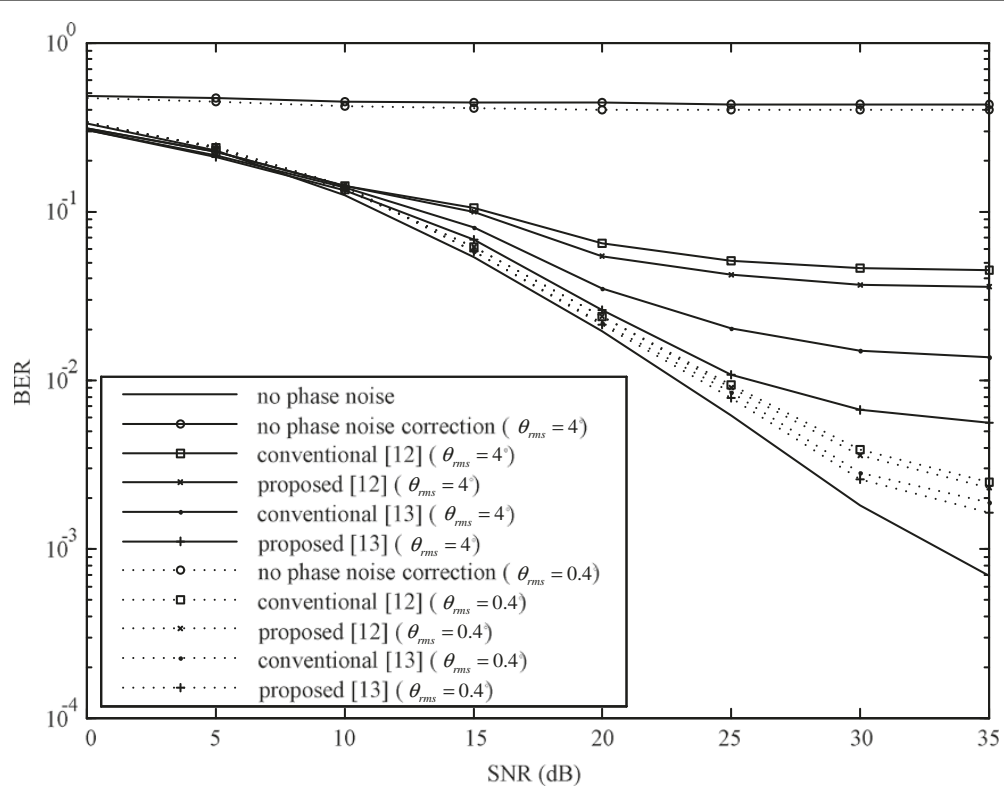

Figure 3 BER performance: stationary phase noise case. BER versus SNR for stationary phase noise, where the standard deviations are $4^{\circ}$ and $0.4^{\circ}$.

However, it can be observed from Figure 2 that for the severe case where phase noise rate is 0.1 (solid lines in Figure 2), the ICI dominates the effect of phase noise. Since [12] only aims to cancel CPE without canceling any of the ICI terms, the performance of "conventional [12]" is not satisfactory. After [12] is preprocessed by our proposed Wiener filter preprocessing algorithm, a performance improvement can be observed from the "proposed
[12]" compared to the "conventional [12]". However, it is observed that the performance gap between the "proposed [12]" and "no phase noise" case is still large, which means that if an ICI-cancelation technique is employed, the performance may be further improved. As can be observed that in this severe phase noise case, the performance of the "proposed [13]" is significantly better than that of the "conventional [13]" for the reason that ICI is

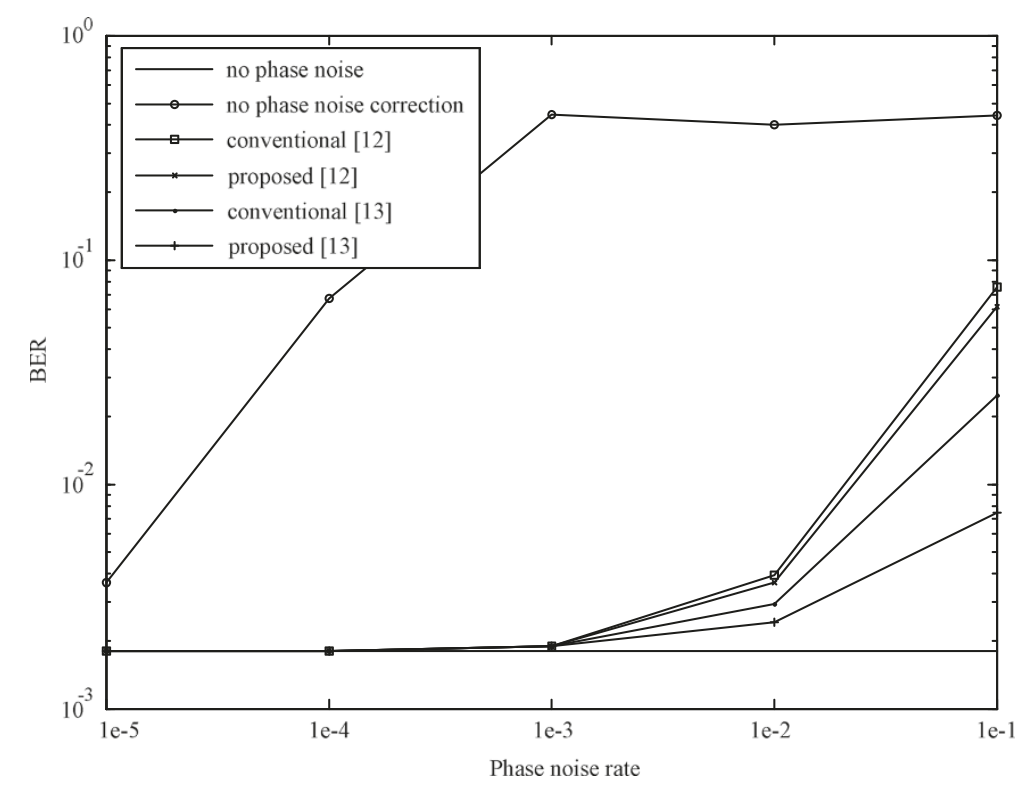

Figure 4 BER performance: nonstationary phase noise case when SNR = $\mathbf{3 0} \mathrm{dB}$. BER versus phase noise rate for nonstationary phase noise, where the range of phase noise rate is from $1 e^{-5}$ to $1 e^{-1}$. 


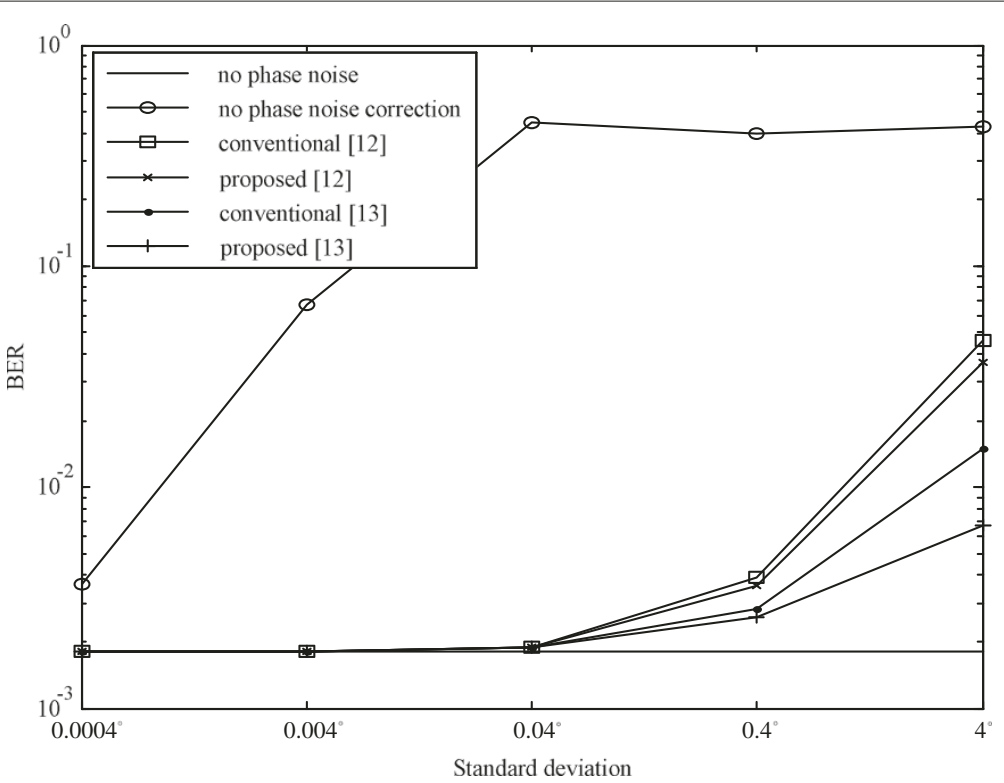

Figure 5 BER performance: stationary phase noise case when SNR $=\mathbf{3 0} \mathrm{dB}$. BER versus standard deviation for stationary phase noise, where the range of standard deviation is from $0.0004^{\circ}$ to $4^{\circ}$.

not negligible and contributes significantly to the overall effect of phase noise. It is noted that ICI can effectively be mitigated and the error floor is obviously lowered through our proposed algorithm.

For the stationary phase noise case, it is generated according to [18] with the standard deviation $\theta_{\text {rms }}=$ $4^{\circ}$ and $0.4^{\circ}$. More specifically, the elements of the correlation matrix for stationary phase noise are given by $\Phi_{\phi(m-n) \phi(m-n)}=\left(\frac{\pi \theta_{\mathrm{rms}}}{180}\right)^{2} e^{-\left(2 \pi \Omega_{0}|m-n| T_{s}\right)}$, where $\Omega_{0}=$ $1000 \mathrm{kHz}$ is the $3-\mathrm{dB}$ bandwidth of a single pole Butterworth filter. It is observed from Figure 3 that similar results can be obtained as the nonstationary phase noise case.

Figures 4 and 5 show the BER performance of the proposed algorithm and that of comparisons $[12,13]$ versus phase noise rate and standard deviation when SNR is set to $30 \mathrm{~dB}$, for the nonstationary and stationary phase noise cases, respectively. The performance advantage of the proposed algorithm over that of conventional $[12,13]$ can be observed especially for severe phase noise cases.

\section{Conclusions}

In this article, we focused on ICI mitigation for OFDM systems in the presence of phase noise. By utilizing the correlation inherently exists in phase noise, a Wiener filter preprocessing algorithm based on the statistics of phase noise has been proposed, which performs directly on the received signal without changing the structure of conventional OFDM systems. Subsequently, the algorithms of previous researches on phase noise cancelation can be performed on the preprocessed received signal. Simulation results showed that for both nonstationary and stationary phase noise cases, the proposed algorithm can effectively mitigate ICI and lower the error floor, and therefore significantly improve the performances of previous researches on phase noise cancelation, especially in the presence of severe phase noise.

\section{Competing interests}

The authors declare that they have no competing interests.

\section{Acknowledgements}

This study was supported in part by the National Science Foundation of China under Grant numbers 61032002, 60902026, and 60972029, the Chinese Important National Science \& Technology Specific Projects under Grant 2011ZX03001-007-01, and by the Program for New Century Excellent Talents in University, NCET-11-0058.

Received: 20 May 2012 Accepted: 24 December 2012

Published: 23 January 2013

\section{References}

1. ETSI TS 101475 V1.2.2, Broad Radio Access Networks (BRAN); HIPERLAN Type 2; Physical (PHY) layer (2001)

2. Digital Video Broadcasting A122, Frame structure channel coding and modulation for a second generation digital terrestrial television broadcasting system (DVB-T2) (2008)

3. IEEE Standard for Local and metropolitan area networks, Part 16: Air interface for fixed and mobile broadband wireless access systems-amendment for physical and medium access control layers for combined fixed and mobile operation in licensed bands (2005)

4. S Sesia, I Toufik, M Baker, LTE-The UMTS Long Term Evolution-From Theory to Practice, 1st edn. (Wiley Press, New York, 2009)

5. D Huang, KB Letaief, Carrier frequency offset estimation for OFDM systems using subcarriers. IEEE Trans. Commun. 54(5), 813-823 (2006)

6. H Abdzadeh-Ziabari, MG Shayesteh, Robust timing frequency synchronization for OFDM systems. IEEE Trans, Veh. Technol. 60(8), 3646-3656 (2011) 
7. M Valkama, M Renfors, V Koivunen, Advanced methods for I/Q imbalance compensation in communication receivers. IEEE Trans. Signal Process. 49(10), 2235-2344 (2001)

8. H Lin, K Yamashita, Subcarrier allocation based compensation for carrier frequency offset and I/Q imbalances in OFDM systems. IEEE Trans. Wirel. Commun. 8(1), 18-23 (2009)

9. T Pollet, MV Bladel, M Moeneclaey, BER sensitivity of OFDM systems to carrier frequency offset and Wiener phase noise. IEEE Trans. Commun 43, 191-193 (1995)

10. L Tomba, On the effect of Wiener phase noise in OFDM systems. IEEE Trans. Commun. 46, 580-583 (1998)

11. AG Armada, Understanding the effects of phase noise in orthogonal frequency division multiplexing (OFDM). IEEE Trans. Broadcast. 47(2), 153-159 (2001)

12. SWu, Y Bar-Ness, OFDM systems in the presence of phase noise: consequences and solutions. IEEE Trans. Commun. 52(11), 1988-1996 (2004)

13. D Petrovic, W Rave, G Fettweis, Effects of phase noise on OFDM systems with and without PLL: characterization and compensation. IEEE Trans. Commun. 55(8), 1607-1616 (2007)

14. P Rabiei, W Namgoong, N Al-Dhahir, A non-iterative technique for phase noise ICI mitigation in packet-based OFDM systems. IEEE Trans. Signal Process. 58(11), 5945-5950 (2010)

15. Q Zou, A Tarighat, AH Sayed, Compensation of phase noise in OFDM wireless systems. IEEE Trans. Signal Process. 55(11), 5407-5424 (2007)

16. Q Zou, A Tarighat, AH Sayed, Joint compensation of IQ imbalance and phase noise in OFDM wireless systems. IEEE Trans. Commun. 57(2), 404-414 (2009)

17. $\mathrm{M}-\mathrm{K}$ Lee, $\mathrm{K}$ Yang, $\mathrm{K}$ Cheun, Iterative receivers based on subblock processing for phase noise compensation in OFDM systems. IEEE Trans. Commun. 59(3), 792-802 (2011)

18. DD Lin, TJ Lim, The variational inference approach to joint data detection and phase noise estimation in OFDM. IEEE Trans. Signal Process. 55(5), 1862-1874 (2007)

19. L Piazzo, P Mandarini, Analysis of phase noise effects in OFDM modems. IEEE Trans. Commun. 50(10), 1696-1705 (2002)

20. DD Lin, RA Pacheco, TJ Lim, D Hatzinakos, Joint estimation of channel response, frequency offset and phase noise in OFDM. IEEE Trans. Signal Process. 54(9), 3542-3554 (2006)

21. S Stefanatos, AK Katsaggelos, Joint data detection channel tracking for OFDM systems with phase noise. IEEE Trans. Signal Process. 56(9), 4230-4243 (2008)

22. J Tao, J Wu, C Xiao, Estimation of channel transfer function and carrier frequency offset for OFDM systems with phase noise. IEEE Trans. Veh. Technol. 58(8), 4380-4387 (2009)

23. H Stark, JW Woods, Probability and Random Processes with Applications to Signal Processing, 3rd edn. (Prentice Hall, New York, 2002)

\section{Submit your manuscript to a SpringerOpen ${ }^{\circ}$ journal and benefit from:}

- Convenient online submission

- Rigorous peer review

- Immediate publication on acceptance

- Open access: articles freely available online

- High visibility within the field

- Retaining the copyright to your article 